\title{
ANCESTRAL GRAPH WITH BIAS IN GENE CONVERSION
}

\author{
SHUHEI MANO, ${ }^{*}$ The Institute of Statistical Mathematics
}

\begin{abstract}
Gene conversion is a genetic mechanism by which one gene is 'copied and pasted' onto another gene, where the direction can be biased between the different types. In this paper, a stochastic model for biased gene conversion within a $d$-unlinked multigene family and its diffusion approximation are developed for a finite Moran population. A connection with a $d$-island model is made. A formula for the fixation probability in the absence of mutation is given. A two-timescale argument is applied in the case of the strong conversion limit. The dual process is generally shown to be a biased voter model, which generates an ancestral bias graph for a given sample. An importance sampling algorithm for computing the likelihood of the sample is deduced.

Keywords: Biased gene conversion; diffusion process; ancestral graph; biased voter model 2010 Mathematics Subject Classification: Primary 60K35
\end{abstract}

Secondary 60J70; 92D15; 92D25

\section{Introduction}

Gene conversion is a mechanism by which a double-strand break in a DNA molecule is repaired using a homologous DNA molecule as a template. As a result, the homologous DNA fragments become identical (i.e. one gene is 'copied and pasted' onto another gene). In diploid organisms, gene conversion can occur between orthologous DNA molecules of paired homologous chromosomes during recombination, referred to as allelic gene conversion. Alternatively, ectopic gene conversion can occur among paralogous DNA molecules of duplicated gene copies in different loci, called a multigene family.

Evolutionary mechanisms are not rigorously tuned. The direction of the conversion appears biased towards $\mathrm{G}$ and $\mathrm{C}$. According to this hypothesis, when an AT versus GC polymorphism exists in homologous DNA molecules, the A or T variant is more likely to be converted to G or $\mathrm{C}$ than the reverse. Regions of a genome that evolve rapidly have been regarded as being under strong positive selection. Surprisingly enough, it was reported that many protein coding changes in the fastest evolving genes of the human genome are not a result of positive selection but a result of biased fixation of AT to GC mutations [1]. In the histone paralogous genes of humans and mice, gene copies that belong to subfamilies with very similar sequences, which are presumably undergoing ectopic gene conversion, have higher GC content than unique gene copies, which are free from ectopic gene conversion [8]. The result of the bias in allelic gene conversion is indistinguishable from that of natural selection, since the models are mathematically identical to each other [17]. In contrast, the dynamics of ectopic gene conversion are poorly understood, although some theoretical models have been developed [18], [23].

In this paper a stochastic model of the dynamics of bias in ectopic gene conversion in a finite population is developed. A corresponding island model of population subdivision [24]

Received 9 January 2012; revision received 31 July 2012.

* Postal address: The Institute of Statistical Mathematics and The Japan Science and Technology Agency, 10-3 Midoricho, Tachikawa, Tokyo 190-8562, Japan. Email address: smano@ism.ac.jp 
with allele-dependent migration, with a diffusive limit identical to that of the model of ectopic gene conversion, is introduced. The model is formulated in terms of a biased voter model [11] on complete graphs, where the interactions between demes are complete and each deme is a complete graph of sites. The biased voter model has a dual process and the limit process generates a random graph for a given sample that is analogous to the coalescent genealogy [12]. We call the graph the ancestral bias graph. The ancestral bias graph is similar to the ancestral selection graph, which was introduced by [13], but the ancestral bias graph is structured with allele-dependent migration. An importance sampling algorithm that can be used to compute the likelihood of a given sample is provided. The algorithm is applied to the mouse histone H2A gene family data set.

\section{The model}

Consider a monoecious panmictic population that consists of $N$ haploid individuals, who have a size $d(\geq 2)$-unlinked multigene family (i.e. duplicated gene copies at unlinked $d$ loci on distinct chromosomes). Assume that the population evolves according to a continuous-time Moran model, in which an individual produces one offspring at a time. The type of the offspring is modified from that of the parent according to mutation and gene conversion mechanisms. The offspring then replaces an individual chosen at random from the population. The offspring may replace its own parent. The replaced individual is removed from the population, keeping the population size constant. We assume that an individual reproduces at a rate of $\lambda_{N}$.

Assume that in the multigene family there are two types of gene: allele $A$ and allele $a$. Let $c(0<c<1)$ be the probability with which a gene at a particular locus in an offspring is converted by a gene of any one of the other $d-1$ loci in the offspring with equal probability. Only a subset of the total conversion events involves different alleles. Among such conversion events involving different alleles, let $(1+b) / 2$ be the fraction of these events that results in an allele $a$ being converted by an allele $A$, and, similarly, let $(1-b) / 2$ be the fraction of the events that result in an allele $A$ being converted by an allele $a$, where $0 \leq b \leq 1$. The conversion event is biased if $b>0$ [18]. The probability with which an allele $a$ is converted by an allele $A$, and an allele $A$ is converted by an allele $a$ is $c(1+b) /(d-1)$ and $c(1-b) /(d-1)$, respectively. For example, when $d=3$, an individual of type $A A a$ produces an offspring of type Aaa, $a A a$, and $A A A$ with probabilities $c(1-b) / 2, c(1-b) / 2$, and $c(1+b)$, respectively. For each locus, an offspring will have the same allelic type as the parent with probability $1-u$ and will have the other type with probability $u$. The coincidence of a gene conversion or a mutation is ignored.

The state of the population at time $t$ can be represented as a continuous-time Markov chain $\boldsymbol{W}^{N}(t)=\left(W_{\alpha}^{N}(t)\right)$, where $W_{\alpha}^{N}(t)$ is the number of individuals of type $\alpha \in\{A, a\}^{d}$ in the population at time $t$. If $\boldsymbol{W}^{N}(t)=\boldsymbol{w}$, the transition to $\boldsymbol{w}+\boldsymbol{e}_{\alpha}-\boldsymbol{e}_{\beta}(\beta \neq \alpha)$ occurs at a rate of

$$
\lambda_{N} w_{\alpha} \frac{w_{\beta}}{N}\left(1-\sum_{\gamma \neq \alpha} q_{\alpha \gamma}\right)+\lambda_{N} \frac{w_{\beta}}{N} \sum_{\gamma \neq \alpha} w_{\gamma} q_{\gamma \alpha},
$$

where $q_{\alpha \beta}$ is the probability with which an individual of type $\alpha$ changes to type $\beta$. For example, $q_{A A a, A a a}=c(1-b) / 2+u(1-u)^{2}$. Other transitions are impossible; for example, $\boldsymbol{w}$ to $\boldsymbol{w} \pm \boldsymbol{e}_{\alpha}$ is impossible since the population size is constant. Then, if we see the $\alpha$ th component, the transition to $w_{\alpha}+1$ occurs at a rate of

$$
\lambda_{N} w_{\alpha} \frac{N-w_{\alpha}}{N}\left(1-\sum_{\gamma \neq \alpha} q_{\alpha \gamma}\right)+\lambda_{N} \frac{N-w_{\alpha}}{N} \sum_{\gamma \neq \alpha} w_{\gamma} q_{\gamma \alpha}
$$


and the transition to $w_{\alpha}-1$ occurs at a rate of

$$
\lambda_{N} w_{\alpha} \frac{w_{\alpha}}{N} \sum_{\gamma \neq \alpha} q_{\alpha \gamma}+\lambda_{N} \frac{w_{\alpha}}{N} \sum_{\gamma \neq \alpha} w_{\gamma}\left(1-q_{\gamma \alpha}\right) .
$$

Let $X_{i}^{N}(t)$ denote the fraction of allele $a$ in the $i$ th locus at time $t$. The limiting diffusion approximation is obtained by setting $\lambda_{N}=N / 2$, assuming that $N u \rightarrow \theta$ and $N c \rightarrow \gamma$ as $N \rightarrow \infty$. Since $\mathbb{E}\left[X_{i}^{N}(1)-x_{i}\right]=m_{i}(\boldsymbol{x})+o(1), \mathbb{E}\left[\left(X_{i}^{N}(1)-x_{i}\right)^{2}\right]=x_{i}\left(1-x_{i}\right)+o(1)$, and $\mathbb{E}\left[\left(X_{i}^{N}(1)-x_{i}\right)^{4}\right]=o(1)$ for $i=1,2, \ldots, d$, where

$$
m_{i}(\boldsymbol{x})=\frac{\gamma^{\prime}}{2}\left(\bar{x}-x_{i}\right)+\frac{\theta}{2}\left(1-2 x_{i}\right)-b \frac{\gamma^{\prime}}{2 d}\left[\left(1-2 x_{i}\right) \sum_{j \neq i} x_{j}+(d-1) x_{i}\right],
$$

$\boldsymbol{X}^{N}(\cdot)$ converges weakly to the limit diffusion in the space of paths $\omega:[0, \infty) \rightarrow[0,1]^{d}[5]$ with generator

$$
\mathcal{L}=\mathscr{L}_{0}-b \mathcal{L}_{1}
$$

where

$$
\begin{aligned}
& \mathcal{L}_{0}=\sum_{i=1}^{d} \frac{x_{i}\left(1-x_{i}\right)}{2} \frac{\partial^{2}}{\partial x_{i}^{2}}+\frac{\gamma^{\prime}}{2} \sum_{i=1}^{d}\left(\bar{x}-x_{i}\right) \frac{\partial}{\partial x_{i}}+\frac{\theta}{2} \sum_{i=1}^{d}\left(1-2 x_{i}\right) \frac{\partial}{\partial x_{i}}, \\
& \mathcal{L}_{1}=\frac{\gamma^{\prime}}{2 d} \sum_{i=1}^{d}\left[\left(1-2 x_{i}\right) \sum_{j \neq i} x_{j}+(d-1) x_{i}\right] \frac{\partial}{\partial x_{i}} .
\end{aligned}
$$

Here, $\bar{x}$ is the arithmetic mean of $\boldsymbol{x}$ and $\gamma^{\prime}=d \gamma /(d-1)$.

It is noteworthy that the generator (2.2) also appears as the diffusive limit of the $d$-island model [24] with allele-dependent migration. The interesting correspondence between a model of a multigene family with gene conversion and a $d$-island model was previously mentioned in [15]. Here, in the current model of a multigene family with biased gene conversion, the corresponding $d$-island model with allele-dependent migration gives a far more intuitive picture of the dual process than the original model of a multigene family (see Section 4).

A $d$-island model is a subdivided population consisting of $d$ demes, where each deme is occupied by $N$ haploid individuals and all pairs of demes can exchange migrants symmetrically. The population evolves according to a continuous-time Moran model, in which an individual produces one offspring at a time. The allelic type of the offspring is modified from that of the parent according to the mutation mechanism. The offspring then replaces an individual chosen at random from the same deme or an individual chosen at random from any of the other demes. The offspring may replace its own parent. The replaced individual is removed from the deme, keeping the deme sizes constant. We assume that an individual reproduces at a rate $\lambda_{N}$ and replaces an individual of the same deme. In addition, during the migration process, alleles $A$ and $a$ replace an individual in another deme at rates $\lambda_{N} \xi_{A}$ and $\lambda_{N} \xi_{a}$, respectively. An offspring will have the same allelic type as the parent with probability $1-u$ and will have the other type with probability $u$. Here $\xi_{A}=c(1+b) /(d-1)$ and $\xi_{a}=c(1-b) /(d-1)$ with $0 \leq b \leq 1$, where $c(0<c<1)$ is the probability of migration. The migration mechanism has allele-dependent bias if $b>0$.

The state of the population at time $t$ can be represented as a continuous-time Markov chain $Z^{N}(t)=\left(Z_{i}^{N}(t)\right)$, where $Z_{i}^{N}(t)$ is the number of individuals of allele $A$ in the $i$ th deme at 
time $t$. If $\boldsymbol{Z}^{N}(t)=\boldsymbol{z}$, the transition to $z+\boldsymbol{e}_{i}$ occurs at a rate of

$$
\lambda_{N}\left(z_{i}+\xi_{A} \sum_{k \neq i} z_{k}\right) \frac{N-z_{i}}{N}(1-u)+\lambda_{N}\left[\left(N-z_{i}\right)+\xi_{a} \sum_{k \neq i}\left(N-z_{k}\right)\right] \frac{N-z_{i}}{N} u
$$

and the transition to $z-\boldsymbol{e}_{i}$ occurs at a rate of

$$
\lambda_{N}\left[\left(N-z_{i}\right)+\xi_{a} \sum_{k \neq i}\left(N-z_{k}\right)\right] \frac{z_{i}}{N}(1-u)+\lambda_{N}\left(z_{i}+\xi_{A} \sum_{k \neq i} z_{k}\right) \frac{z_{i}}{N} u
$$

Let $X_{i}(t)=Z_{i}(t) / N$ denote the frequency of allele $A$ in the $i$ th deme at time $t$. The limiting diffusion approximation is obtained by setting $\lambda_{N}=N / 2$, assuming that $N u \rightarrow \theta$ and $N c \rightarrow \gamma$ as $N \rightarrow \infty \cdot \boldsymbol{X}^{N}(\cdot)$ converges weakly to the limit diffusion in $[0,1]^{d}$, whose generator is identical to the generator of the model of biased gene conversion in a multigene family (2.2).

\section{Fixation probability}

The fate of a single mutant is important in molecular evolutionary problems. By using a birth-death process, an expression of the fixation probability of a single mutant in the weak conversion limit $(\gamma \rightarrow 0$, where $\gamma=N c$ ) was obtained [23]. In contrast, when $\gamma$ is large, the effects of bias upon the fixation probability can be significant, as was observed recently using computer simulations [15]. However, no analytical expression has been obtained.

In this section the mutation rate is set to 0 (i.e. $u=0$ ). A path of the continuous-time Moran model will eventually be absorbed into either of the absorbing states $\mathbf{0}$ and $\mathbf{1}$, since without mutation allele $A$ cannot recover in a population fixed by allele $a$, and vice versa. For the diffusive limit of the continuous-time Moran model, we have the following lemma.

Lemma 3.1. The extremal stationary states of the diffusive limit of the continuous-time Moran model taking values in $[0,1]^{d}$ and governed by generator (2.2) are $\delta_{\mathbf{0}}$ and $\delta_{\mathbf{1}}$.

Proof. Let $v$ be a extremal stationary state. The system of equations for $\mu_{\boldsymbol{a}}^{\infty}=\left\langle v, \boldsymbol{X}^{\boldsymbol{a}}\right\rangle$, where $\boldsymbol{X}^{\boldsymbol{a}}=\prod_{i=1}^{d} X_{i}^{a_{i}}$ and $\langle\nu, f\rangle=\int v(\mathrm{~d} x) f(x)$, is obtained by applying Itô's formula to $\boldsymbol{X}^{\boldsymbol{a}}$ with generator (2.2). That is,

$$
\begin{aligned}
& \left\{\sum_{i=1}^{d} \frac{a_{i}\left(a_{i}-1\right)}{2}+\frac{\gamma}{2}(1+b) \sum_{i=1}^{d} a_{i}\right\} \mu_{\boldsymbol{a}}^{\infty} \\
& \quad=\sum_{i=1}^{d} \frac{a_{i}\left(a_{i}-1\right)}{2} \mu_{\boldsymbol{a}-\boldsymbol{e}_{i}}^{\infty}+\frac{\gamma^{\prime}}{d} \sum_{i<j} a_{i}\left\{(1-b) \mu_{\mathbf{a}-\boldsymbol{e}_{i}+\boldsymbol{e}_{j}}^{\infty}+2 b \mu_{\boldsymbol{a}+\boldsymbol{e}_{j}}^{\infty}\right\}
\end{aligned}
$$

This implies that $\mu_{\boldsymbol{a}}^{\infty}=\mu_{\boldsymbol{e}_{1}}^{\infty}$ for all $\boldsymbol{a} \neq \mathbf{0}$. Thus, we have $v=\mu_{\boldsymbol{e}_{1}}^{\infty} \delta_{\mathbf{1}}+\left(1-\mu_{\boldsymbol{e}_{1}}^{\infty}\right) \delta_{\mathbf{0}}$.

Theorem 3.1. For the diffusive limit of the continuous-time Moran model, the fixation probability of allele a with $\boldsymbol{X}(\mathbf{0})=\boldsymbol{p}$ is

$$
\pi(\boldsymbol{p})=\bar{p}-\left\{(d-1)\left[\bar{p}+\bar{p}(1-\bar{p}) \gamma^{\prime}\right]+\frac{2}{d} \sum_{i<j} p_{i} p_{j}\right\} b+O\left(b^{2}\right) .
$$


Proof. Let $\mu_{\boldsymbol{a}}(t)=\mathbb{E}\left[\boldsymbol{X}(t)^{\boldsymbol{a}}\right]$. By Lemma 3.1 and Lebesgue's dominated convergence theorem, $\lim _{t \rightarrow \infty} \mu_{\boldsymbol{a}}(t)=\mu_{\boldsymbol{e}_{1}}^{\infty}=\pi(\boldsymbol{p})$. Consider the expansion of the Laplace transform of the moments $\mu_{\boldsymbol{a}}(t)$ as a power series in $b: \tilde{\mu}_{\boldsymbol{a}}(s)=\tilde{\mu}_{\boldsymbol{a}}^{(0)}(s)+\tilde{\mu}_{\boldsymbol{a}}^{(1)}(s) b+\cdots$. At the zeroth order in $b$ we have the system of equations

$$
\left(s+\gamma^{\prime}\right) \tilde{\mu}_{\boldsymbol{e}_{i}}^{(0)}-\frac{\gamma^{\prime}}{d} \sum_{j=1}^{d} \tilde{\mu}_{\boldsymbol{e}_{j}}^{(0)}=p_{i}, \quad i=1,2, \ldots, d,
$$

with solution

$$
\tilde{\mu}_{\boldsymbol{e}_{i}}^{(0)}(s)=\frac{\bar{p}}{s}+\frac{p_{i}-\bar{p}}{s+\gamma^{\prime}}, \quad i=1,2, \ldots, d .
$$

By applying the inverse Laplace transform, we have $\mu_{\boldsymbol{e}_{1}}^{(0)}=\bar{p}+\left(p_{1}-\bar{p}\right) \mathrm{e}^{-\gamma^{\prime} t}$. In the same manner, for $i=1,2, \ldots, d$,

$$
\left(\frac{s}{2}+1+\gamma^{\prime}\right) \tilde{\mu}_{2 \boldsymbol{e}_{i}}^{(0)}-\tilde{\mu}_{\boldsymbol{e}_{i}}^{(0)}-\frac{\gamma^{\prime}}{d} \sum_{j=1}^{n} \tilde{\mu}_{\boldsymbol{e}_{i}+\boldsymbol{e}_{j}}^{(0)}=\frac{p_{i}^{2}}{2},
$$

and, for $i \neq j, i, j=1,2, \ldots, d$,

$$
\left(s+2 \gamma^{\prime}\right) \tilde{\mu}_{\boldsymbol{e}_{i}+\boldsymbol{e}_{j}}^{(0)}-\frac{\gamma^{\prime}}{d} \sum_{k=1}^{n}\left(\tilde{\mu}_{\boldsymbol{e}_{j}+\boldsymbol{e}_{k}}^{(0)}+\tilde{\mu}_{\boldsymbol{e}_{i}+\boldsymbol{e}_{k}}^{(0)}\right)=p_{i} p_{j} .
$$

These equations can be solved for $\tilde{\mu}_{2 \boldsymbol{e}_{i}}^{(0)}$ and $\tilde{\mu}_{\boldsymbol{e}_{i}+\boldsymbol{e}_{j}}^{(0)}$. At the first order in $b$, we have the following system of equations: for $i=1,2, \ldots, d$,

$$
\left(s+\gamma^{\prime}\right) \tilde{\mu}_{\boldsymbol{e}_{i}}^{(1)}-\frac{\gamma^{\prime}}{d} \sum_{j=1}^{n} \tilde{\mu}_{\boldsymbol{e}_{j}}^{(1)}=\frac{\gamma^{\prime}}{d}\left\{(d-2) \tilde{\mu}_{\boldsymbol{e}_{i}}^{(0)}+\sum_{j=1}^{d} \tilde{\mu}_{\boldsymbol{e}_{j}}^{(0)}-2 \sum_{j(\neq i)} \tilde{\mu}_{\boldsymbol{e}_{i}+\boldsymbol{e}_{j}}^{(0)}\right\} .
$$

Then

$$
\tilde{\mu}_{\boldsymbol{e}_{i}}^{(1)}(s)=\frac{a_{0}}{s}+\sum_{j=1}^{d} \frac{a_{j}}{s-s_{j}}, \quad i=1,2, \ldots, d,
$$

where

$$
a_{0}=(d-1) \bar{p}\left\{1+\gamma^{\prime}(1-\bar{p})\right\}-\frac{2}{d} \sum_{i<j} p_{i} p_{j} .
$$

Here the $s_{j}(<0)$ are eigenvalues of generator (2.2) and the $a_{j \neq 0}$ are constants independent of $s$. Then, by applying the inverse Laplace transform, the lemma follows.

Remark 3.1. In the weak conversion limit $(\gamma \rightarrow 0)$, the expression for the fixation probability (3.1) with $\boldsymbol{p}=\boldsymbol{e}_{1} / N$ in large $N$ agrees with Equation (8) of [23], which was obtained by a different method.

Remark 3.2. It may seem curious that the effects of bias (linear term in $b$ ) do not vanish in the weak conversion limit $(\gamma \rightarrow 0$ ). Of course, the linear term disappears without gene conversion $(c=0)$. If gene conversion is extremely weak, all loci are monomorphic except for very short periods of time when a single locus is segregating. An allele fixes in the polymorphic locus and after a long period of time biased gene conversion creates another polymorphic locus. The process continues until all loci are fixed by the same allele. Since the locus-by-locus spreading is biased, the bias is effective even when gene conversion is extremely weak (see [23]). 
When $b=1$, all conversion events involving different alleles result in an allele $a$ being converted by an allele $A$.

Theorem 3.2. When $b=1$,

$$
\pi(\boldsymbol{p})=\left\{1-(1-\bar{p}) d \gamma+O\left(\gamma^{2}\right)\right\} \prod_{i=1}^{d} p_{i} .
$$

Proof. The fixation probability satisfies the Kolmogorov backward equation

$$
\left(\mathscr{L}_{0}-\mathscr{L}_{1}\right) \pi(\boldsymbol{x})=0
$$

with $\pi(\mathbf{0})=0$ and $\pi(\mathbf{1})=1$. Assuming that $\pi(\boldsymbol{x})=\sum_{\boldsymbol{a}} c_{\boldsymbol{a}} \boldsymbol{x}^{\boldsymbol{a}}$, (3.2) gives $c_{\mathbf{1}}=1-d \gamma+$ $O\left(\gamma^{2}\right), c_{\mathbf{1}+\boldsymbol{e}_{i}}=\gamma c_{\mathbf{1}}$, and $c_{\boldsymbol{a}}=O\left(\gamma^{2}\right)$ for $\boldsymbol{a} \neq \mathbf{1}, \mathbf{1}+\boldsymbol{e}_{i}$, where $i=1,2, \ldots, d$.

\section{Strong conversion limit}

Let us define the strong conversion limit with allele-dependent migration as $d N b \rightarrow \beta$ as $N \rightarrow \infty$. Interestingly, the strong conversion limit of the continuous-time Moran model of biased gene conversion within a $d$-unlinked multigene family has a limiting diffusion whose generator is identical to that of the very fundamental one-locus diffusion with directional selection. Various results known for the process also hold for the strong conversion limit of the biased gene conversion model.

By applying the singular perturbation theory, Ethier and Nagylaki [6] obtained a diffusion approximation of Markov chains with two time scales. Consider the continuous-time Moran model for the $d$-island population subdivision with allele-dependent migration, whose transition rates are given by (2.3) and (2.4) with $\lambda_{N}=1$. Consider the mean of the frequencies $\bar{X}^{N}(t)=\sum_{i=1}^{d} X_{i}^{N}(t) / d$ and the deviations from the mean $Y_{i}^{N}(t)=X_{i}^{N}(t)-\bar{X}^{N}(t), i=$ $1,2, \ldots, d$. Set $\varepsilon_{N}^{-1}=d N / 2 /(1+c)$ and $\delta_{N}^{-1}=1$. Asymptotically, as $N \rightarrow \infty$, the infinitesimal variances and means of $\bar{X}^{N}\left(\cdot / \varepsilon_{N}\right)$ are

$$
\begin{gathered}
\varepsilon_{N}^{-1} \mathbb{E}\left[\bar{X}^{N}(1)-\bar{x}\right]=m(\bar{x}, \boldsymbol{y})+o(1), \\
\varepsilon_{N}^{-1} \mathbb{E}\left[\left(\bar{X}^{N}(1)-\bar{x}\right)^{2}\right]=v(\bar{x}, \boldsymbol{y})+o(1),
\end{gathered}
$$

where

$$
\begin{gathered}
m(\bar{x}, \boldsymbol{y})=\frac{\beta c}{1+c}\left\{\bar{x}-\frac{d}{d-1}\left[\bar{x}^{2}-\frac{1}{d^{2}} \sum_{i=1}^{d}\left(y_{i}+\bar{x}\right)^{2}\right]\right\}+\frac{d \theta}{2}(1-2 \bar{x}) \\
v(\bar{x}, \boldsymbol{y})=\bar{x}-\frac{c}{1+c} \frac{d}{d-1} \bar{x}^{2}+\frac{c-d+1}{(1+c) d(d-1)} \sum_{i=1}^{d}\left(y_{i}+\bar{x}\right)^{2}
\end{gathered}
$$

Those of $\boldsymbol{Y}^{N}\left(\cdot / \delta_{N}\right)$ are

$$
\delta_{N}^{-1} \mathbb{E}\left[\boldsymbol{Y}^{N}(1)-\boldsymbol{y}\right]=\boldsymbol{f}(\bar{x}, \boldsymbol{y})+o(1),
$$

where $\boldsymbol{f}(\bar{x}, \boldsymbol{y})=-d c \boldsymbol{y} /(d-1)$. Also,

$$
\varepsilon_{N}^{-1} \mathbb{E}\left[\left(\bar{X}^{N}(1)-\bar{x}\right)^{4}\right]=o(1), \quad \delta_{N}^{-1} \operatorname{var}\left(y^{N}(1)\right)=o(1) .
$$


According to Theorem 3.3 of [6], under conditions (4.1)-(4.4) and using the fact that the zero solution of

$$
\delta_{\infty}^{-1}[\boldsymbol{Y}(k+1, \bar{x}, \boldsymbol{y})-\boldsymbol{Y}(k, \bar{x}, \boldsymbol{y})]=\boldsymbol{f}(\bar{x}, \boldsymbol{Y}(k, \bar{x}, \boldsymbol{y})), \quad \boldsymbol{Y}(0, \bar{x}, \boldsymbol{y})=\boldsymbol{y},
$$

for $k=0,1, \ldots$ is globally asymptotically stable, $\boldsymbol{Y}^{N}\left(t / \varepsilon_{N}\right) \rightarrow \mathbf{0}$ in probability for every $t>0$, and $\bar{X}^{N}\left(\cdot / \varepsilon_{N}\right)$ converges weakly to a diffusion process on the surface $\boldsymbol{y}=\mathbf{0}$.

Theorem 4.1. The continuous-time Moran model for the d-island population subdivision with allele-dependent migration whose transition rates are given by (2.3) and (2.4) has the limiting diffusion of strong migration in $[0,1]$ with generator

$$
\frac{\bar{x}(1-\bar{x})}{2} \frac{\partial^{2}}{\partial \bar{x}^{2}}-\left[\frac{\beta c}{1+c} \bar{x}(1-\bar{x})-\frac{d \theta}{2}(1-2 \bar{x})\right] \frac{\partial}{\partial \bar{x}} .
$$

This generator also appears as the strong conversion limit of the continuous-time Moran model of biased gene conversion within a d-unlinked multigene family with transition rates (2.1).

Remark 4.1. The generator (4.5) is identical to that of the diffusion process of the onelocus, two-allele model with directional selection and selection intensity $2 \beta c /(1+c)$. In strong migration limits of population subdivision considered in [16], the effects of population subdivision disappear and the panmictic diffusion holds if the migration is conservative. In contrast, in the strong conversion limit of the continuous-time Moran model of biased gene conversion, the effects of a multigene structure remain as an effective selection.

Remark 4.2. The continuous-time Moran model for one-locus, two-alleles with directional selection can be formulated using the biased voter model and the dual has a limit process that generates the ancestral selection graph [13]. The continuous-time Moran model of biased gene conversion has an analogue of the ancestral selection graph, which we call the ancestral bias graph (see Section 5). In the strong conversion limit, the process generating the ancestral bias graph should converge into the process generating the ancestral selection graph with selection intensity $2 \beta c /(1+c)$. A direct proof of this observation without using the duality argument seems difficult.

\section{Ancestral bias graph}

The above introduced $d$-island model with allele-dependent migration also has a formulation in terms of the biased voter model on a set of complete graphs. Denote by $\boldsymbol{I}=\left(I_{i}\right), I_{i}=$ $\{1,2, \ldots, N\}, i=1,2, \ldots, d$, sets of sites, where $I_{i}$ is the set of sites in the $i$ th graph. The biased voter model is a continuous-time Markov process whose state at time $t$ is denoted by $\eta_{t}: \boldsymbol{I} \rightarrow\{A, a\}$. If $x \in I_{i}$, and $\eta_{t}(x)=A$ or $\eta_{t}(x)=a$, then $x$ is occupied by an individual of allelic type $A$ or, respectively, $a$ at time $t$. The process $\left\{\eta_{t} ; t \geq 0\right\}$ evolves according to the following rules.

(i) For $x=1,2, \ldots, N$ and $i=1,2, \ldots, d$, the individual at $x \in I_{i}$ produces an offspring at rate $\lambda_{N}$ within $I_{i}$.

(ii) The offspring has the same allelic type as the parent with probability $1-u$ and has the other type with probability $u$.

(iii) For $x=1,2, \ldots, N$ and $i, j=1,2, \ldots, d, j \neq i$, the individual at $x \in I_{i}$ produces an offspring in $I_{j}$ at rates depending on the allelic type. If $\eta_{t}(x)=A$, the rate is $\lambda_{N} \xi_{A}$; if $\eta_{t}(x)=a$, the rate is $\lambda_{N} \xi_{a}$. Furthermore, $\xi_{A}-\xi_{a}=2 c b /(d-1)$. 
(iv) At the time when the birth event occurs, one of the $N$ sites is chosen at random and the individual at this site is replaced by the offspring. The offspring is allowed to replace its own parent.

This process can be visualized as a percolation process [4], [10], and the construction is similar to that of the continuous-time Moran model with selection [13]. The idea is to construct the process using a collection of independent Poisson processes by drawing arrows on the spacetime coordinate system $\boldsymbol{I} \times[0, \infty)$. These arrows indicate where and when the offspring is produced and sent. We begin by connecting arrows to each timeline at the times of arrivals in a Poisson process that describes the birth process. For each $(x, y) \in I_{i}^{2}, i=1,2, \ldots, d$, let $\left\{W_{i, s}^{x, y} ; s \geq 1\right\}$ denote the times of arrivals in a Poisson process with rate $\lambda_{N} / N$. For each $(x, y) \in I_{i} \times I_{j}, i, j=1,2, \ldots, d, i \neq j$, let $\left\{Z_{i, j, s}^{x, y} ; s \geq 1\right\}$ denote the times of arrivals in a Poisson process with rate $\lambda_{N} \xi_{A} / N$. Let $\left\{U_{i, j, s}^{x, y} ; s \geq 1\right\}$ and $\left\{V_{i, s}^{x, y} ; s \geq 1\right\}$, $i, j=1,2, \ldots, d, i \neq j$, be sequences of independent, uniformly distributed random variables in $(0,1)$. For times $W_{i, s}^{x, y}$, we draw an arrow from $x \in I_{i}$ to $y \in I_{i}$ to indicate the birth of an offspring at $x$ that is sent to $y$. For times $Z_{i, j, s}^{x, y}$, we draw an arrow from $x \in I_{i}$ to $y \in I_{j}$ to indicate the birth of an offspring at $x$ that is then sent to $y$. If $U_{i, j, s}^{x, y}<\xi_{a} / \xi_{A}$, we place a ' $\delta$ ' at the tip of the arrow; otherwise, we label the arrow with a ' 2 '. In other words, we have $\delta$-arrows and 2-arrows entering a site $y$ at rates $\lambda_{N} \xi_{a}$ and $\lambda_{N}\left(\xi_{A}-\xi_{a}\right)$, respectively. Then, the following rule will apply: type- $A$ individuals can give birth through both types of arrow, but type- $a$ individuals can only give birth through $\delta$-arrows. The process $\left\{V_{i, s}^{x, y} ; s \geq 1\right\}$ is used as the mutation process; if $V_{i, s}^{x, y}<u$, a mutation occurs. We represent a mutation event by filled circles on the arrows. A realization of the percolation diagram in the case $d=2$ and $N=4$ is shown in Figure 1. Here $I_{1}$ and $I_{2}$ are the left and the right graphs, respectively. If the set of sites occupied by type- $a$ individuals is initially $\{1\} \in I_{2}$ then, at time $t$, the set of sites occupied by type- $a$ individuals is $\{2\} \in I_{1}$ and $\{3\} \in I_{2}$. The paths of the $a$ s are indicated by thick lines. By reversing time, the ancestral history of individuals at sites are followed and, thus, their types are determined. The resulting process is called the dual process. A realization of the dual process, which was obtained from Figure 1 by simply reversing time and the direction of the arrows, is shown in Figure 2. Here, the ancestral history of a sample consists of individuals at sites $\{1,2\} \in I_{1}$ and $\{1\} \in I_{2}$ at dual time 0 , indicated by thick lines.

Consider the dual process of a sample of size $\boldsymbol{n}=\left(n_{i}\right), i=1,2, \ldots, d$, at time 0 taken from a population, when the mutation rate is set to 0 (i.e. $u=0$ ). Assume that there are $\boldsymbol{k}$ particles in the limiting process. A coalescing event occurs when a particle crosses an unmarked arrow and lands on the site of a different particle contained in the dual process. This occurs at rates

$$
\lambda_{N} k_{i} \frac{k_{i}-1}{N}=\frac{k_{i}\left(k_{i}-1\right)}{2}, \quad i=1,2, \ldots, d .
$$

A migration event occurs when a particle crosses a $\delta$-arrow. This occurs at a rate of

$$
\lambda_{N} \xi_{a} n_{i} \frac{N-k_{j}}{N} \rightarrow \frac{(1-b) \gamma}{2(d-1)} k_{i} \quad \text { as } N \rightarrow \infty
$$

for $i, j=1,2, \ldots, d, j \neq i$. A branching event occurs when a particle crosses a 2-arrow. This occurs at a rate of

$$
\lambda_{N}\left(\xi_{A}-\xi_{a}\right) k_{i} \frac{N-k_{j}}{N} \rightarrow \frac{b \gamma}{d-1} k_{i} \quad \text { as } N \rightarrow \infty
$$

for $i, j=1,2, \ldots, d, i \neq j$. The original particle continues along the continuing path, and 


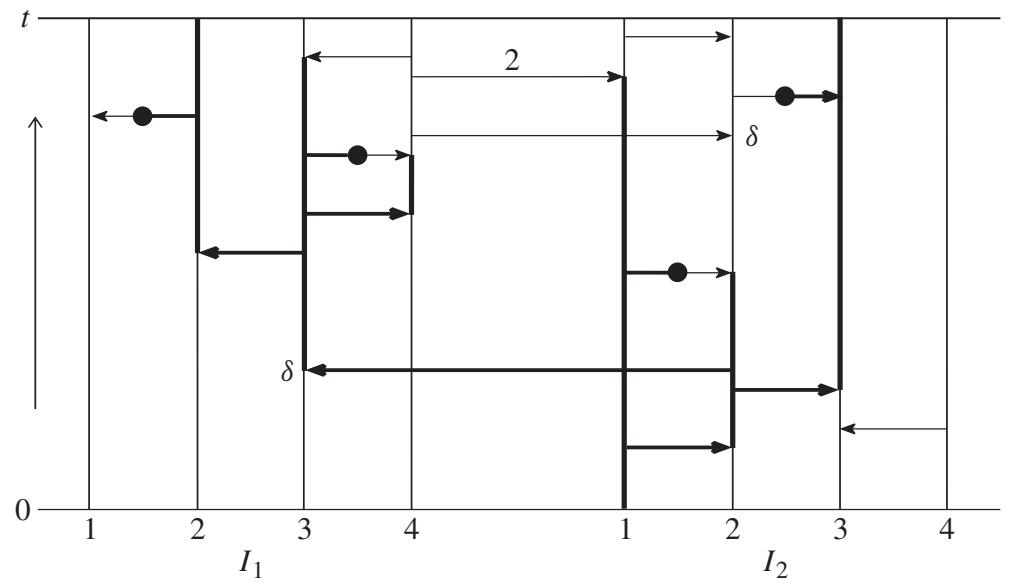

FIGURE 1: A graphical representation of the biased voter model for the case $d=2$ and $N=4$. If, initially, the set of $a$ s is $\{1\} \in I_{2}$ then at time $t$ the set of $a$ s is $\{2\} \in I_{1}$ and $\{3\} \in I_{2}$. The paths of the $a$ are indicated by thick lines.

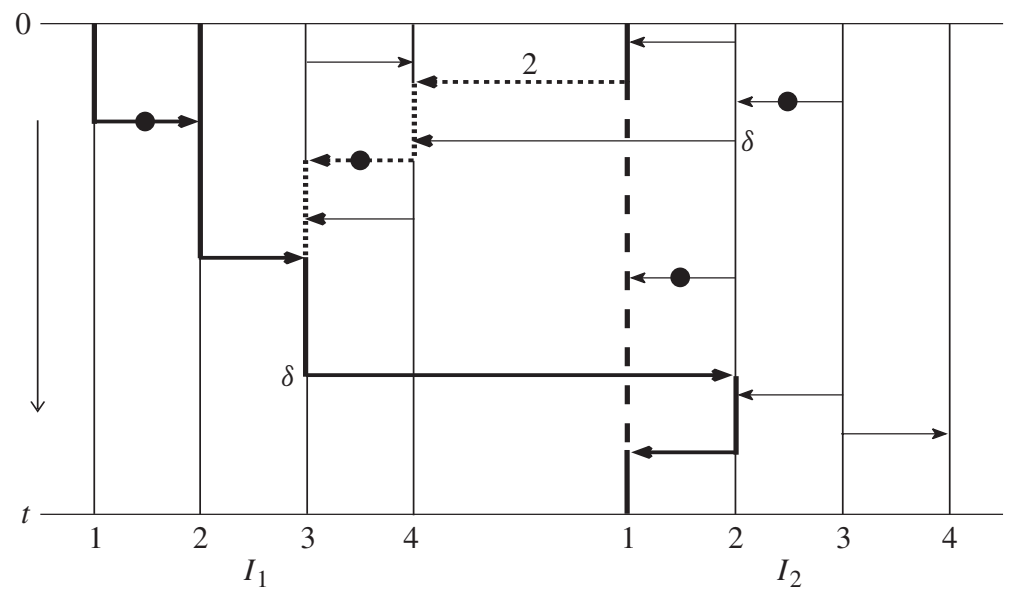

Figure 2: A graphical representation of the dual process of the biased voter model. The ancestral history of a sample consists of individuals at sites $\{1,2\} \in I_{1}$ and $\{1\} \in I_{2}$ at dual time 0 , indicated by thick lines. The ultimate ancestor is in $\{1\} \in I_{2}$, at dual time $t$. If the ultimate ancestor is $a$ then the true genealogy contains the dotted line and does not contain the dashed line.

the new particle that arose from the branching follows the 2-arrow (incoming path). The new particle can land on a site that is already contained in the dual process. The event, which was called collision in [13], occurs with probability $k_{j} / N$, and can be ignored in the limit $N \rightarrow \infty$. An analogue of the coalescent genealogy can be obtained by rescaling time and the parameters as $\lambda_{N}=N / 2, \xi_{A}=c(1+b) /(d-1)$, and $\xi_{a}=c(1-b) /(d-1)$ with $N c \rightarrow \gamma$ as $N \rightarrow \infty$. We call the limiting process $\left\{g_{n}(t) ; t \geq 0\right\}$ the ancestral bias graph, which consists of three components: the set-valued process $\left\{\mathcal{A}_{n, i}(t) ; t \geq 0, i=1,2, \ldots, d\right\}$, the jump process $\left\{R_{m} ; m \geq 1\right\}$, and the label process $\left\{\left(\beta_{m}, \gamma_{m}\right) ; m \geq 1\right\}$. Here $\mathcal{A}_{\boldsymbol{n}, i}(t)$ is the set of particles in the $i$ th deme at time $t, R_{m}$ is the time of the $m$ th event, and $\beta_{m}$ and $\gamma_{m}$ denote branched particles or coalesced particles at the $m$ th event, respectively. 
Let the size process $\boldsymbol{A}_{\boldsymbol{n}}(t)=\left(\left|\mathcal{A}_{n, i}(t)\right|\right), i=1,2, \ldots, d$. Then $\left\{\boldsymbol{A}_{\boldsymbol{n}}(t) ; t \geq 0\right\}$ is a $d$-dimensional birth and death process with rates $(5.1)-(5.3)$ in the state space $\mathbb{Z}_{+}^{d}$. The process is similar to the process that was introduced in [21] to analyze stationary states and the stability of the stepping stone model involving directional selection. By either a direct application of Itô's formula or using rules to generate an ancestral bias graph for a sample that consists of allele $a$, we obtain the following duality relation. The proofs are essentially the same as the proof of Theorem 2.1 of [14] for the size process of the ancestral selection graph.

Lemma 5.1. The moment dual of the birth and death process $\boldsymbol{A}_{\boldsymbol{n}}(t)$ is the Wright-Fisher diffusion $\boldsymbol{X}(t)$ in $[0,1]^{d}$ governed by generator (2.2):

$$
\mathbb{E}_{p}\left[X(t)^{n}\right]=\mathbb{E}_{n}\left[\boldsymbol{p}^{A_{n}(t)}\right]
$$

Corollary 5.1. The joint probability generating function of the stationary measure of the birth and death process $\left(\pi_{A}\right)$ with rates $(5.1)-(5.3)$ is

$$
\mathbb{E}_{\pi_{A}}\left[p^{A}\right]=\pi(p)
$$

where $\pi(\boldsymbol{p})$ is the fixation probability given in Theorem 3.1. In particular,

$$
\begin{aligned}
\pi_{A}\left(\boldsymbol{e}_{i}\right) & =\frac{1}{d}-b\left(\frac{d-1}{d}-\gamma\right)+O\left(b^{2}\right), \\
\pi_{A}\left(2 \boldsymbol{e}_{i}\right) & =b \frac{\gamma}{d}+O\left(b^{2}\right), \\
\pi_{A}\left(\boldsymbol{e}_{i}+\boldsymbol{e}_{j}\right) & =b \frac{2}{d}(1+\gamma)+O\left(b^{2}\right),
\end{aligned}
$$

for $i, j=1,2, \ldots, d, i \neq j$. Other configurations have probabilities of order $O\left(b^{2}\right)$.

If the process hits $\left|\boldsymbol{A}_{\boldsymbol{n}}(t)\right|=1$ for the first time, we call the particle at that time ultimate ancestor. When $0<b<1$, the ultimate ancestor always exists because the state space is irreducible. In contrast, when $b=1$, the ultimate ancestor can never exist as long as $\#\left\{i ; n_{i} \geq 1\right\} \geq 2$. The state space is reducible and states $n_{i}=0$ for some $i$ are transient. Therefore, if $n_{i}=0$ for some $i, \pi_{A}(\boldsymbol{n})=0$. Moreover, the fixation probability given in Theorem 3.2 gives the following result.

Corollary 5.2. When $b=1$,

$$
\pi_{A}(\mathbf{1})=1-d \gamma+O\left(\gamma^{2}\right), \quad \pi_{A}\left(\mathbf{1}+\boldsymbol{e}_{i}\right)=\gamma \pi_{A}(\mathbf{1}) \text { for } i=1,2, \ldots, d .
$$

Other configurations have probabilities of order $O\left(\gamma^{2}\right)$.

Theorem 5.1. Let $W_{\boldsymbol{n}}$ be the waiting time to the ultimate ancestor of a sample of $\boldsymbol{n}$ genes. Then $\mathbb{E}\left[W_{\boldsymbol{e}_{1}}\right]=0$ and

$$
\begin{aligned}
r(\boldsymbol{n}) \mathbb{E}\left[W_{\boldsymbol{n}}\right]= & \sum_{i=1}^{d} n_{i}\left(n_{i}-1\right) \mathbb{E}\left[W_{\boldsymbol{n}-\boldsymbol{e}_{i}}\right]+\sum_{i \neq j} \frac{2 b \gamma n_{i}}{d-1} \mathbb{E}\left[W_{\boldsymbol{n}+\boldsymbol{e}_{j}}\right] \\
& +\sum_{i \neq j} \frac{(1-b) \gamma n_{i}}{d-1} \mathbb{E}\left[W_{\boldsymbol{n}-\boldsymbol{e}_{i}+\boldsymbol{e}_{j}}\right]+2,
\end{aligned}
$$

where $r(\boldsymbol{n})=\sum_{i=1}^{d} n_{i}\left\{n_{i}-1+\gamma(1+b)\right\}$. 
Proof. This is clear by considering the waiting time until the first event in the birth and death process with rates (5.1)-(5.3).

Corollary 5.3. For a sample of size 2,

$$
\begin{aligned}
\mathbb{E}\left[W_{2 \boldsymbol{e}_{i}}\right]= & d+b \frac{2(d \gamma)^{3}+(d-1)\left\{8(d \gamma)^{2}+\left(10 d^{2}+7 d-2\right) \gamma+2(d-1)(2 d-1)\right\}}{3 \gamma\{d \gamma+2(d-1)\}}, \\
& +O\left(b^{2}\right), \\
\mathbb{E}\left[W_{\boldsymbol{e}_{i}+\boldsymbol{e}_{j}}\right]= & d+\frac{d-1}{\gamma}+b \frac{2 d^{2} \gamma^{3}+2(d-1)\left\{3 d^{2} \gamma+2\left(d^{2}-1\right)\right\}}{3 \gamma\{d \gamma+2(d-1)\}}+O\left(b^{2}\right),
\end{aligned}
$$

for $i, j=1,2, \ldots, d, i \neq j$.

Consider superimposing the mutation process, denoted by $\left\{y_{i} ; t \geq 0, i=1,2, \ldots, d\right\}$, on the ancestral bias graph. The rate is $\lambda_{N} u k_{i} \rightarrow \theta k_{i} / 2$ as $N \rightarrow \infty$ for $i=1,2, \ldots, n$. Depending on the type of the ultimate ancestor and the mutation events along the branches, certain parts of the ancestral bias graph may not be accessible to individuals since only individuals of allelic type $A$ may cross 2-arrows. In Figure 2, if the allelic type of the ultimate ancestor is $a$ then the true genealogy contains the dotted line and does not contain the dashed line. In contrast, if the allelic type of the ultimate ancestor is $A$, then the true genealogy contains the dashed line. To simulate the joint distribution of a sample of size $\boldsymbol{n}$ from a large population that is in equilibrium, we proceed as follows.

(i) Construct an ancestral bias graph starting with $\boldsymbol{n}$ particles, until the first time it reaches the ultimate ancestor.

(ii) Choose the ultimate ancestor's type for the sample according to the stationary measure of the diffusion process governed by generator (2.2) (see Section 6).

(iii) Run the mutation process forward along the ancestral bias graph starting at the ultimate ancestor.

In step (iii), the type of particle that continues after the meeting of incoming and continuing branches (branching event in the dual process) is identical to Table 2 of [13], in which types ' 1 ' and ' 2 ' are ' $a$ ' and 'A', respectively.

As for the ancestral selection graph [13], the effects of bias on the ancestral bias graph when imposing the mutation process are insignificant when the mutation rate is very large or very small.

Lemma 5.2. Denote the time to the most recent common ancestor by $T_{\mathrm{MRCA}}$. When $\theta=0$, the distribution of $T_{\mathrm{MRCA}}$ does not depend on $b$ and is identical to that of the $d$-island model of population subdivision without bias. When $\theta \gg b \gamma$, the distribution of $T_{\mathrm{MRCA}}$ is also identical to the model without bias.

Proof. The proof is essentially the same as that of Theorem 3.12 of [13].

\section{Sampling distributions}

Suppose that $\boldsymbol{n}$ genes are sampled from a population. A sample configuration is denoted by $\left(\boldsymbol{n}_{a}, \boldsymbol{n}_{A}\right)$, where $\boldsymbol{n}=\boldsymbol{n}_{a}+\boldsymbol{n}_{A}$, and $n_{a, i}$ and $n_{A, i}$ are the numbers of allele $a$ and allele $A$ in the 
$i$ th deme, respectively. Let $p(\boldsymbol{n})$ be the multinomial sampling distribution, or the likelihood, of a sample of $\boldsymbol{n}$ genes taken from a population in equilibrium:

$$
p(\boldsymbol{n})=\mathbb{E}_{\pi_{X}}\left[\prod_{i=1}^{d} \frac{n_{i} !}{n_{a, i} ! n_{A, i} !} X_{i}^{n_{a, i}}\left(1-X_{i}\right)^{n_{A, i}}\right] .
$$

Theorem 6.1. The sampling distribution $p(\boldsymbol{n})$ satisfies

$$
\begin{aligned}
r(\boldsymbol{n}) p(\boldsymbol{n})= & \sum_{i=1}^{d}\left[\left(n_{a, i}-1\right) n_{i} p\left(\boldsymbol{n}-\boldsymbol{e}_{a, i}\right)+\left(n_{A, i}-1\right) n_{i} p\left(\boldsymbol{n}-\boldsymbol{e}_{A, i}\right)\right] \\
& +\theta \sum_{i=1}^{d}\left[\left(n_{A, i}+1\right) p\left(\boldsymbol{n}_{a}-\boldsymbol{e}_{a, i}+\boldsymbol{e}_{A, i}\right)+\left(n_{a, i}+1\right) p\left(\boldsymbol{n}+\boldsymbol{e}_{a, i}-\boldsymbol{e}_{A, i}\right)\right] \\
& +\frac{(1-b) \gamma^{\prime}}{d} \sum_{i, j \neq i} n_{i}\left[\frac{n_{a, j}+1}{n_{j}+1} p\left(\boldsymbol{n}-\boldsymbol{e}_{a, i}+\boldsymbol{e}_{a, j}\right)\right. \\
& +\frac{2 b \gamma^{\prime}}{d} \sum_{i, j \neq i}\left[\frac{n_{A, i}\left(n_{A, j}+1\right)}{n_{j}+1} p\left(\boldsymbol{n}+\boldsymbol{e}_{A, j}\right)+\frac{n_{i}\left(n_{a, j}+1\right)}{n_{j}+1} p\left(\boldsymbol{n}+\boldsymbol{e}_{A, i}+\boldsymbol{e}_{A, j}\right)\right] \\
& \left.+\frac{\left(n_{a, i}+1\right)\left(n_{A, j}+1\right)}{n_{j}+1} p\left(\boldsymbol{n}+\boldsymbol{e}_{a, i}-\boldsymbol{e}_{A, i}+\boldsymbol{e}_{A, j}\right)\right],
\end{aligned}
$$

where $r(\boldsymbol{n})=\sum_{i=1}^{d} n_{i}\left\{n_{i}-1+\theta+\gamma(1+b)\right\}$. The probabilities with negative arguments are 0 . The boundary condition is

$$
p\left(\boldsymbol{e}_{a, i}\right)=\rho, \quad p\left(\boldsymbol{e}_{A, i}\right)=1-\rho, \quad i=1,2, \ldots, d .
$$

Proof. The proof follows by a direct application of Itô's formula to the moments in (6.1). It can also be proved via the rules used to generate the ancestral bias graph when imposing the mutation process [13].

Remark 6.1. In the weak mutation limit $(\theta \rightarrow 0), p\left(\boldsymbol{n}_{a}\right)=p\left(\boldsymbol{e}_{1}\right)=\rho$ for any nonzero $\boldsymbol{n}_{a} \in$ $\operatorname{span}\left\{\boldsymbol{e}_{a, 1}, \boldsymbol{e}_{a, 2}, \ldots, \boldsymbol{e}_{a, d}\right\}, p\left(\boldsymbol{0}, \boldsymbol{n}_{A}\right)=1-\rho$ for any nonzero $\boldsymbol{n}_{A} \in \operatorname{span}\left\{\boldsymbol{e}_{A, 1}, \boldsymbol{e}_{A, 2}, \ldots, \boldsymbol{e}_{A, d}\right\}$, and $p(\boldsymbol{n})=0$ for any nonzero $\boldsymbol{n} \notin\left\{\operatorname{span}\left\{\boldsymbol{e}_{a, 1}, \boldsymbol{e}_{a, 2}, \ldots, \boldsymbol{e}_{a, d}\right\}, \operatorname{span}\left\{\boldsymbol{e}_{A, 1}, \boldsymbol{e}_{A, 2}, \ldots, \boldsymbol{e}_{A, d}\right\}\right\}$, because the sample allelic type is solely determined by the allelic type of the ultimate ancestor. For example, if a sample of size 2 is taken from the $i$ th deme, $p\left(2 \boldsymbol{e}_{a, i}\right)+p\left(2 \boldsymbol{e}_{A, i}\right)=1$ and $p\left(\boldsymbol{e}_{a, i}+\boldsymbol{e}_{A, i}\right)=0$.

Corollary 6.1. For a sample of size 1 ,

$$
p\left(\boldsymbol{e}_{a, i}\right)=\frac{1}{2}-\frac{\left(1+2 \theta+\gamma^{\prime}\right) \gamma}{2\left\{\gamma^{\prime} / d+2 \theta\left(1+2 \theta+\gamma^{\prime}\right)\right\}} b+O\left(b^{2}\right), \quad i=1,2, \ldots, d,
$$

and, for a sample of size 2 ,

$$
\begin{gathered}
p\left(2 \boldsymbol{e}_{a, i}\right)=\frac{\gamma^{\prime} / d+\theta+\left(1+2 \theta+\gamma^{\prime}\right)(\theta-b \gamma)}{2\left\{\gamma^{\prime} / d+2 \theta\left(1+2 \theta+\gamma^{\prime}\right)\right\}}+O\left(b^{2}\right), \\
p\left(\boldsymbol{e}_{a, i}+\boldsymbol{e}_{a, j}\right)=\frac{\gamma^{\prime} / d+\left(1+2 \theta+\gamma^{\prime}\right)(\theta-b \gamma)}{2\left\{\gamma^{\prime} / d+2 \theta\left(1+2 \theta+\gamma^{\prime}\right)\right\}}+O\left(b^{2}\right),
\end{gathered}
$$




$$
\begin{gathered}
p\left(\boldsymbol{e}_{a, i}+\boldsymbol{e}_{A, i}\right)=\frac{\theta\left(2 \theta+\gamma^{\prime}\right)}{\gamma^{\prime} / d+2 \theta\left(1+2 \theta+\gamma^{\prime}\right)}+O\left(b^{2}\right), \\
p\left(\boldsymbol{e}_{a, i}+\boldsymbol{e}_{A, j}\right)=\frac{\theta\left(1+2 \theta+\gamma^{\prime}\right)}{2\left\{\gamma^{\prime} / d+2 \theta\left(1+2 \theta+\gamma^{\prime}\right)\right\}}+O\left(b^{2}\right),
\end{gathered}
$$

for $i, j=1,2, \ldots, d, i \neq j$. We obtain $p\left(2 \boldsymbol{e}_{A, i}\right)$ and $p\left(\boldsymbol{e}_{A, i}+\boldsymbol{e}_{A, j}\right)$ from (6.2) and (6.3), respectively, by replacing $b$ with $(-b)$. These expressions reduce to those in the model without bias when $\theta \gg b \gamma$ (see Lemma 5.2). The effects of bias (linear term in b) vanish in the weak conversion limit $(\gamma \rightarrow 0)$ (cf. Remark 3.2), but the effects do not vanish in the weak mutation limit (cf. Lemma 5.2).

Remark 6.2. The identity coefficients in a multigene family were defined by [19]. For unlinked loci, the average probability of identity at the same locus is $p\left(2 \boldsymbol{e}_{a, i}\right)+p\left(2 \boldsymbol{e}_{A, i}\right)$, and that at different loci of the same or homologous chromosomes is $p\left(\boldsymbol{e}_{a, i}+\boldsymbol{e}_{a, j}\right)+p\left(\boldsymbol{e}_{A, i}+\boldsymbol{e}_{A, j}\right)$. When $b=0$, these expressions reduce to Equations (12) of [19].

\section{Importance sampling}

The state space of the ancestral histories of a sample is huge and closed-form expressions for the likelihood are not available. Griffiths and Tavaré [9] introduced an importance sampling method on the ancestral process back in time. Stephens and Donnelly [22] constructed an efficient proposal distribution, and De Iorio and Griffiths [2] characterized the proposal distribution in terms of the generator of the dual diffusion process, which describes the population gene frequencies. De Iorio and Griffiths [3] applied the method to construct an importance sampling algorithm for computing the likelihood of samples in subdivided population models.

A history $\left\{H_{k} ; k=0,-1, \ldots,-m\right\}$ is defined as the set of ancestral configurations at the embedded events in the Markov process where coalescence, migration, branching, and mutation events take place. Here $H_{0}$ denotes the current state (sample configuration), and $H_{-m}$ denotes the state when an ultimate ancestor is reached $\left(\boldsymbol{e}_{A, i}\right.$ or $\left.\boldsymbol{e}_{a, i}\right)$. The system of equations given in Theorem 6.1 is written as $p\left(H_{k}\right)=\sum_{\left\{H_{k-1}\right\}} p\left(H_{k} \mid H_{k-1}\right) p\left(H_{k-1}\right)$. The importance sampling representation is based on finding a good approximation to the reverse chain probabilities $\hat{p}\left(H_{k-1} \mid H_{k}\right)$. The importance sampling representation is then

$$
p\left(H_{0}\right)=\mathbb{E}_{\hat{p}}\left[\frac{p\left(H_{0} \mid H_{-1}\right)}{\hat{p}\left(H_{-1} \mid H_{0}\right)} \cdots \frac{p\left(H_{-m+1} \mid H_{-m}\right)}{\hat{p}\left(H_{-m} \mid H_{-m+1}\right)} p\left(H_{-m}\right)\right] \approx \frac{1}{M} \sum_{i=1}^{M} \frac{p\left(\mathscr{H}^{(i)}\right)}{\hat{p}\left(\mathscr{H}^{(i)}\right)} \hat{p}\left(H_{0}\right),
$$

where $\mathbb{E}_{\hat{p}}$ denotes the expectation taken over histories in the reverse direction with the reverse chain transition probabilities $\hat{p}\left(H_{k-1} \mid H_{k}\right)$, and $\mathscr{H}^{(1)}, \mathscr{H}^{(2)}, \ldots, \mathscr{H}^{(M)}$ are independent sample paths from the reverse chain.

From (6.1), it follows that

$$
\pi(\alpha \mid i, \boldsymbol{n}) p(\boldsymbol{n})=\mathbb{E}_{\pi_{X}}\left[\left(X_{i} \delta_{\alpha, a}+\left(1-X_{i}\right) \delta_{\alpha, A}\right) q_{\boldsymbol{n}}(\boldsymbol{X})\right]
$$

and

$$
\begin{aligned}
& \pi\left(\beta \mid j, \boldsymbol{n}+\boldsymbol{e}_{a, i} \delta_{\alpha, a}+\boldsymbol{e}_{A, i} \delta_{\alpha, A}\right) \pi(\alpha \mid i, \boldsymbol{n}) p(\boldsymbol{n}) \\
& \quad=\mathbb{E}_{\pi_{X}}\left[\left(X_{j} \delta_{\beta, a}+\left(1-X_{j}\right) \delta_{\beta, A}\right)\left(X_{i} \delta_{\alpha, a}+\left(1-X_{i}\right) \delta_{\alpha, A}\right) q_{\boldsymbol{n}}(\boldsymbol{X})\right],
\end{aligned}
$$


where

$$
q_{\boldsymbol{n}}(\boldsymbol{x})=\prod_{i=1}^{d} \frac{n_{i} !}{n_{a, i} ! n_{A, i} !} x_{i}^{n_{a, i}}\left(1-x_{i}\right)^{n_{A, i}}
$$

and $\pi(\alpha \mid i, \boldsymbol{n})$ is the probability that an additional gene taken from deme $i$ is of allelic type $\alpha$ given that we have a configuration $\boldsymbol{n}$. Assume that (7.2) and (7.3) hold for an approximate sampling distribution $\hat{p}(\boldsymbol{n})$ obtained by setting [3]

$$
\mathbb{E}_{\pi_{X}}\left[\mathcal{L}_{i} \frac{\partial}{\partial X_{i}} q_{\boldsymbol{n}}(\boldsymbol{X})\right]=0
$$

where

$$
\mathcal{L}_{i}=\frac{x_{i}\left(1-x_{i}\right)}{2} \frac{\partial}{\partial x_{i}}+\frac{\gamma^{\prime}}{2}\left(\bar{x}-x_{i}\right)+\frac{\theta}{2}\left(1-2 x_{i}\right)-b \frac{\gamma^{\prime}}{2 d}\left[\left(1-2 x_{i}\right) \sum_{j \neq i} x_{j}+(d-1) x_{i}\right],
$$

yielding a system of equations for $\hat{\pi}(\alpha \mid i, \boldsymbol{n})$, which is an approximation of $\pi(\alpha \mid i, \boldsymbol{n})$ for $\alpha=a, A$ and $i=1,2, \ldots, d$. That is, we obtain

$$
\begin{aligned}
r_{i} \hat{\pi}(a \mid i, \boldsymbol{n})= & n_{a, i}+\theta+\frac{(1-b) \gamma^{\prime}}{d} \sum_{j \neq i} \hat{\pi}(a \mid j, \boldsymbol{n}) \\
& +\frac{2 b \gamma^{\prime}}{d} \frac{n_{A, i}\left(n_{i}+1\right)}{n_{a, i}+1} \hat{\pi}(a \mid i, \boldsymbol{n}) \sum_{j \neq i} \hat{\pi}\left(A \mid j, \boldsymbol{n}+\boldsymbol{e}_{a, i}\right)
\end{aligned}
$$

and

$$
\begin{aligned}
& r_{i} \hat{\pi}(A \mid i, \boldsymbol{n})=n_{A, i}+\theta+ \frac{(1-b) \gamma^{\prime}}{d} \sum_{j \neq i} \hat{\pi}(A \mid j, \boldsymbol{n}) \\
&+\frac{2 b \gamma^{\prime}}{d} \sum_{j \neq i}\left[\frac{\left(n_{i}+1\right)^{2}}{n_{A, i}+1} \hat{\pi}(A \mid i, \boldsymbol{n}) \hat{\pi}\left(a \mid j, \boldsymbol{n}+\boldsymbol{e}_{A, i}\right)\right. \\
&\left.+\left(n_{i}+1\right) \hat{\pi}(a \mid i, \boldsymbol{n}) \hat{\pi}\left(A \mid j, \boldsymbol{n}+\boldsymbol{e}_{a, i}\right)\right],
\end{aligned}
$$

where $r_{i}=n_{i}+2 \theta+\gamma$. The system has the solution

$$
\hat{\pi}(\alpha \mid i, \boldsymbol{n})=\left(\frac{n_{\alpha, i}+\theta}{\gamma^{\prime} / d}+\sum_{j=1}^{d} \frac{n_{\alpha, j}-n_{\alpha, i}}{\gamma^{\prime} / d+r_{j}}\right)\left(\frac{\gamma^{\prime} / d+r_{i}}{\gamma^{\prime} / d}-\sum_{j=1}^{d} \frac{\gamma^{\prime} / d+r_{i}}{\gamma^{\prime} / d+r_{j}}\right)^{-1}+O(b) .
$$

When $b=0,(7.6)$ is the exact probability that an additional gene taken from deme $i$ is of allelic type $\alpha$ given that we have a configuration $\boldsymbol{n}$. Even for $b>0$, solving the quadratic system of equations (7.4) and (7.5) is computationally expensive. Using expression (7.6) with $b=0$ and renormalizing the proposal distribution is practically useful.

From Bayes' rule, $p\left(H_{k-1} \mid H_{k}\right)=p\left(H_{k} \mid H_{k-1}\right) p\left(H_{k-1}\right) / p\left(H_{k}\right)$, but the $p\left(H_{k-1}\right) / p\left(H_{k}\right)$ are unknown. The importance sampling proposal distribution is obtained by substituting $\hat{\pi}$ for $\pi$ in $p\left(H_{k-1}\right) / p\left(H_{k}\right)$, with the importance weights given by

$$
\frac{p\left(H_{k} \mid H_{k-1}\right)}{\hat{p}\left(H_{k-1} \mid H_{k}\right)}=\frac{\hat{p}\left(H_{k}\right)}{\hat{p}\left(H_{k-1}\right)}
$$


For example, in the case $H_{k}=\boldsymbol{n}$ and $H_{k-1}=\boldsymbol{n}+\boldsymbol{e}_{a, i}-\boldsymbol{e}_{A, i}+\boldsymbol{e}_{A, j}$, the proposal distribution is

$$
\begin{aligned}
\hat{p}\left(H_{k-1} \mid H_{k}\right)= & p\left(H_{k} \mid H_{k-1}\right) \frac{\hat{p}\left(\boldsymbol{n}+\boldsymbol{e}_{a, i}-\boldsymbol{e}_{A, i}+\boldsymbol{e}_{A, j}\right)}{\hat{p}\left(\boldsymbol{n}-\boldsymbol{e}_{A, i}\right)} \frac{\hat{p}\left(\boldsymbol{n}-\boldsymbol{e}_{A, i}\right)}{\hat{p}(\boldsymbol{n})} \\
= & \frac{2 b \gamma^{\prime}}{d} \frac{\left(n_{a, i}+1\right)\left(n_{A, j}+1\right)}{r(\boldsymbol{n})\left(n_{j}+1\right)} \\
& \times \frac{n_{i}\left(n_{j}+1\right)}{\left(n_{a, i}+1\right)\left(n_{A, j}+1\right)} \hat{\pi}\left(A \mid j, \boldsymbol{n}+\boldsymbol{e}_{a, i}-\boldsymbol{e}_{A, i}\right) \hat{\pi}\left(a \mid i, \boldsymbol{n}-\boldsymbol{e}_{A, i}\right) \\
& \times \frac{n_{A, i}}{n_{i} \hat{\pi}\left(A \mid i, \boldsymbol{n}-\boldsymbol{e}_{A, i}\right)} \\
= & \frac{2 b \gamma n_{A, i} \hat{\pi}\left(A \mid j, \boldsymbol{n}+\boldsymbol{e}_{a, i}-\boldsymbol{e}_{A, i}\right) \hat{\pi}\left(a \mid i, \boldsymbol{n}-\boldsymbol{e}_{A, i}\right)}{(n-1) r(\boldsymbol{n}) \hat{\pi}\left(A \mid i, \boldsymbol{n}-\boldsymbol{e}_{A, i}\right)},
\end{aligned}
$$

and the importance weight is

$$
\frac{\left(n_{a, i}+1\right)\left(n_{A, j}+1\right) \hat{\pi}\left(A \mid i, \boldsymbol{n}-\boldsymbol{e}_{A, i}\right)}{n_{A, i}\left(n_{j}+1\right) \hat{\pi}\left(A \mid j, \boldsymbol{n}+\boldsymbol{e}_{a, i}-\boldsymbol{e}_{A, i}\right) \hat{\pi}\left(a \mid i, \boldsymbol{n}-\boldsymbol{e}_{A, i}\right)} .
$$

The proposal distribution and respective importance weights for the other cases are summarized in Table 1.

In (7.1) $p\left(H_{-m}\right)$ is equal to either $\rho$ or $1-\rho$. A closed-form expression for $\rho$ is not available, but it is easily obtained by using the perfect simulation (coupling from the past) [7], [20]. For the perfect simulation, a parent-independent mutation model, where an allele mutates to $A$ and $a$ with equal probability conditional on a mutation occurring with mutation rate $2 u$, is useful. The parent-independent mutation model is probabilistically equivalent to the mutation model discussed in previous sections, but the treatment in the perfect simulation becomes much simpler.

TABLe 1.

\begin{tabular}{ccc}
\hline$H_{k-1}$ & Proposal distribution & Importance weight \\
\hline $\boldsymbol{n}-\boldsymbol{e}_{a, i}$ & $\frac{n_{a, i}\left(n_{a, i}-1\right)}{r(\boldsymbol{n}) \hat{\pi}\left(a \mid i, \boldsymbol{n}-\boldsymbol{e}_{a, i}\right)}$ & $\frac{n_{i}}{n_{a, i}} \hat{\pi}\left(a \mid i, \boldsymbol{n}-\boldsymbol{e}_{a, i}\right)$ \\
$\boldsymbol{n}-\boldsymbol{e}_{A, i}$ & $\frac{n_{A, i}\left(n_{A, i}-1\right)}{r(\boldsymbol{n}) \hat{\pi}\left(A \mid i, \boldsymbol{n}-\boldsymbol{e}_{A, i}\right)}$ & $\frac{n_{i}}{n_{A, i}} \hat{\pi}\left(A \mid i, \boldsymbol{n}-\boldsymbol{e}_{A, i}\right)$ \\
$\boldsymbol{n}-\boldsymbol{e}_{a, i}+\boldsymbol{e}_{A, i}$ & $\frac{\theta n_{a, i} \hat{\pi}\left(A \mid i, \boldsymbol{n}-\boldsymbol{e}_{a, i}\right)}{r(\boldsymbol{n}) \hat{\pi}\left(a \mid i, \boldsymbol{n}-\boldsymbol{e}_{a, i}\right)}$ & $\frac{\left(n_{A, i}+1\right) \hat{\pi}\left(a \mid i, \boldsymbol{n}-\boldsymbol{e}_{a, i}\right)}{n_{a, i} \hat{\pi}\left(A \mid i, \boldsymbol{n}-\boldsymbol{e}_{a, i}\right)}$ \\
$\boldsymbol{n}+\boldsymbol{e}_{a, i}-\boldsymbol{e}_{A, i}$ & $\frac{\theta n_{A, i} \hat{\pi}\left(a \mid i, \boldsymbol{n}-\boldsymbol{e}_{A, i}\right)}{r(\boldsymbol{n}) \hat{\pi}\left(A \mid i, \boldsymbol{n}-\boldsymbol{e}_{A, i}\right)}$ & $\frac{\left(n_{a, i}+1\right) \hat{\pi}\left(A \mid i, \boldsymbol{n}-\boldsymbol{e}_{A, i}\right)}{n_{A, i} \hat{\pi}\left(a \mid i, \boldsymbol{n}-\boldsymbol{e}_{A, i}\right)}$ \\
$\boldsymbol{n}-\boldsymbol{e}_{a, i}+\boldsymbol{e}_{a, j}$ & $\frac{(1-b) \gamma^{\prime} n_{a, i} \hat{\pi}\left(a \mid j, \boldsymbol{n}-\boldsymbol{e}_{a, i}\right)}{d r(\boldsymbol{n}) \hat{\pi}\left(a \mid i, \boldsymbol{n}-\boldsymbol{e}_{a, i}\right)}$ & $\frac{n_{i}\left(n_{a, i}+1\right) \hat{\pi}\left(a \mid i, \boldsymbol{n}-\boldsymbol{e}_{a, i}\right)}{n_{a, i}\left(n_{j}+1\right) \hat{\pi}\left(a \mid j, \boldsymbol{n}-\boldsymbol{e}_{a, i}\right)}$ \\
$\boldsymbol{n}-\boldsymbol{e}_{A, i}+\boldsymbol{e}_{A, j}$ & $\frac{(1-b) \gamma^{\prime} n_{A, i} \hat{\pi}\left(A \mid j, \boldsymbol{n}-\boldsymbol{e}_{A, i}\right)}{d r(\boldsymbol{n}) \hat{\pi}\left(A \mid i, \boldsymbol{n}-\boldsymbol{e}_{A, i}\right)}$ & $\frac{n_{i}\left(n_{A, j}+1\right) \hat{\pi}\left(A \mid i, \boldsymbol{n}-\boldsymbol{e}_{A, i}\right)}{n_{A, i}\left(n_{j}+1\right) \hat{\pi}\left(A \mid j, \boldsymbol{n}-\boldsymbol{e}_{A, i}\right)}$ \\
$\boldsymbol{n}+\boldsymbol{e}_{a, j}$ & $\frac{2 b \gamma^{\prime} n_{i} \hat{\pi}(a \mid j, \boldsymbol{n})}{d r(\boldsymbol{n})}$ & $\frac{n_{a, j}+1}{\left(n_{j}+1\right) \hat{\pi}(a \mid j, \boldsymbol{n})}$ \\
$\boldsymbol{n}+\boldsymbol{e}_{A, j}$ & $\frac{2 b \gamma^{\prime} n_{A, i} \hat{\pi}(A \mid j, \boldsymbol{n})}{d r(\boldsymbol{n})}$ & $\frac{n_{A, j}+1}{\left(n_{j}+1\right) \hat{\pi}(A \mid j, \boldsymbol{n})}$ \\
\hline
\end{tabular}


Consider simulating a sample of size 1 from the ancestral bias graph. We can prove the following theorem on the expected number of events until the ancestral bias graph couples for a sample of size 1 .

Theorem 7.1. Consider the strong conversion limit $(d N b \rightarrow \beta$ as $N \rightarrow \infty)$. Let $T$ be an exponential random variable with rate $\theta / 4$. Let $M$ be the number of events in an ancestral bias graph initiated with a single branch, up to time $T$ in the past. The expected number of events until the ancestral bias graph couples is bounded above by $\mathbb{E}[M]$. Furthermore,

$$
\lim _{\beta \rightarrow \infty} \mathbb{E}\left[\frac{M}{\beta^{2}}\right] \leq \frac{(4 c)^{2}}{\theta} .
$$

Proof. For the first part, the proof is the same as that of Theorem 1 of [7]. For the second part, according to Remark 4.2, the problem is reduced to finding a bound for the expected number of events until the ancestral selection graph with selection intensity $2 \beta c /(1+c)$ initiated with a single branch couples. Theorem 1 of [7] gives the required result.

\section{Example: mouse histone gene family}

Exon sequences of members of a mouse histone H2A gene family (single exon gene and 393 base pairs in length), which consists of 20 gene copies distributed at six unlinked loci in the mouse genome, were retrieved from Ensembl release 63 (see http://www.ensembl.org/). The GC content at the third codon position was $90.2 \%$, which is significantly higher than average GC content in the mouse genome. The substitution rate was estimated to be $2.0 \times 10^{-9}$ per site per generation, noting that the sequence divergence between the mouse sequence and the homologous rat sequence at the third codon position is $16 \%$, assuming that the mouse-rat divergence time is 20 million years and the average generation time is 0.5 years. If substitution occurs symmetrically among nucleotides, two-thirds of the substitutions occur between AT and GC. Under neutrality, the mutation rate between AT and GC is $u=1.3 \times 10^{-9}$. The actual process of conversion involves a piece of a gene. Here, a nucleotide site was considered to be a unit of conversion and linkage among them was also ignored. Nucleotides of the third codon position were analyzed, where AT and GC nucleotides are regarded as allelic types $a$ and $A$, respectively. Assuming that the data set comes from $d=20$ loci of a single chromosome and the maximum likelihood estimate of $\theta, \gamma$, and $b$, which jointly maximize the composite likelihood of the 131 nucleotide sites, was obtained by using the importance sampling algorithm introduced in Section 7. The estimates were $\hat{\theta}=0.0008, \hat{\gamma}=0.04$, and $\hat{b}=0.07$. Then, the conversion rate per site per nucleotide would be $\hat{c}=6.5 \times 10^{-8}$.

\section{Acknowledgements}

The author thanks Brian Charlesworth for suggesting the subject of this paper. He also thanks Robert Griffiths for comments on importance sampling, Tokuzo Shiga for comments in connection with Sections 3 and 5, and Tomoko Ohta and Kai Zeng for comments on ectopic gene conversion, and the anonymous referee for suggestions for improving the presentation.

\section{References}

[1] Berglund, J, Pollard, K. S. and Webster, M. T. (2009). Hotspots of biased nucleotide substitutions in human genes. PLoS Biol. 7, e1000026, 18 pp.

[2] De Iorio, M. ANd Griffiths, R. C. (2004). Importance sampling on coalescent histories. I. Adv. Appl. Prob. 36, 417-433. 
[3] De Iorio, M. And Griffiths, R. C. (2004). Importance sampling on coalescent histories. II. Subdivided population models. Adv. Appl. Prob. 36, 434-454.

[4] Donnelly, P. (1984). The transient behavior of the Moran model in population genetics. Math. Proc. Camb. Phil. Soc. 95, 349-358.

[5] Ethier, S. N. And Kurtz, T. G. (1986). Markov Processes. John Wiley, New York.

[6] Ethier, S. N. And NagYlaki, T. (1980). Diffusion approximations of Markov chains with two time scales and applications to population genetics. Adv. Appl. Prob. 12, 14-49.

[7] Fearnhead, P. (2001). Perfect simulation from population genetic models with selection. Theoret. Pop. Biol. 59, 263-279.

[8] Galtier, N. (2003). Gene conversion drives GC content evolution in mammalian histones. Trends Genet. 19, 65-68.

[9] Griffiths, R. C. And Tavaré, S. (1994). Simulating probability distribution in the coalescent. Theoret. Pop. Biol. 46, 131-159.

[10] Harris, T. E. (1972). Nearest-neighbor Markov interaction process on multidimensional lattices. Adv. Math. 9, 66-89.

[11] Harris, T. E. (1976). On a class of set-valued Markov processes. Ann. Prob. 4, 175-194.

[12] Kingman, J. F. C. (1982). The coalescent. Stoch. Process. Appl. 13, 235-248.

[13] Krone, S. M. and Neuhauser, C. (1997). Ancestral process with selection. Theoret. Pop. Biol. 51, $210-237$.

[14] Mano, S. (2009). Duality, ancestral and diffusion processes in models with selection. Theoret. Pop. Biol. 75, $164-175$.

[15] Mano, S. And Innan, H. (2008). The evolutionary rate of duplicated genes under concerted evolution. Genetics 180, 493-505.

[16] NagYlaKI, T. (1980). The strong-migration limit in geographically structured populations. J. Math. Biol. 9, 101-114.

[17] Nagylaki, T. (1983). Evolution of a finite population under gene conversion. Proc. Nat. Acad. Sci. USA 80, 6278-6281.

[18] Nagylaki, T. and Petes, T. D. (1982). Interchromosomal gene conversion and the maintenance of sequence homogeneity among repeated genes. Genetics 100, 315-337.

[19] Онта, T. (1982). Allelic and nonallelic homology of a supergene family. Proc. Nat. Acad. Sci. USA 79, 3251-3254.

[20] Propp, J. G. AND Wilson, D. B. (1996). Exact sampling with coupled Markov chains and applications to statistical mechanics. Random Struct. Algorithms 9, 223-252.

[21] Shiga, T. and Uchiyama, K. (1986). Stationary states and their stability of the stepping stone model involving mutation and selection. Prob. Theoret. Relat. Fields 73, 87-117.

[22] Stephens, M. And Donnelly, P. (2000). Inference in molecular population genetics. J. R. Statist. Soc. B 62, 605-655.

[23] Walsh, J. B. (1985). Interaction of selection and biased gene conversion in a multigene family. Proc. Nat. Acad. Sci. USA 82, 153-157.

[24] Wright, S. (1951). The genetical structure of populations. Ann. Eugenics 15, 323-354. 